\title{
HYPERTHYROIDISM AND RADIOIODINE
}

Patients with iodine-induced subclinical and clinical hyperthyroidism should be carefully medicated to normalize thyroid function before radioiodine treatment for multinodular goiters, according to a Brazilian research group.

The study by Romão et al. was designed to establish the efficacy of recombinant human $\mathrm{TSH}$ ( $\mathrm{rhTSH}$ ) as an adjuvant to radioiodine therapy for the treatment of patients with multinodular goiters with or without hyperthyroidism. The investigators enrolled 42 patients with multinodular goiters, of whom six had symptoms of clinical hyperthyroidism, 18 had subclinical hyperthyroidism and 18 had normal thyroid function.

Romão et al. first removed iodine from the patients' diet for 90 days before radioiodine therapy. Moreover, the six patients with clinical hyperthyroidism received a daily dose of $10 \mathrm{mg}$ methimazole for 30 days. The treatment protocol comprised a $0.1 \mathrm{mg}$ intramuscular injection of $\mathrm{rTSH}$, followed by administration of $1.1 \mathrm{GBq}$ of radioiodine - a much lower dose than the doses of radioiodine used in previous studies-after $24 \mathrm{~h}$.

After reduction of thyroid volume with radioidine, patients with hyperthyroidism had more adverse effects than euthyroid patients, for example, a very high surge of thyroid hormones in the first 2-3 weeks after treatment. "This could be considered as potentially dangerous owing to the well-known cardiac effects of increased circulating levels of thyroid hormones," comments senior investigator Geraldo Medeiros-Neto (University of São Paulo, Brazil).

The researchers recommend that patients with multinodular goiters and hyperthyroidism be treated with $15 \mathrm{mg}$ methimazole per day for 2 months in combination with a low-iodine diet before radioiodine treatment to avoid the risks of cardiac events.

\section{Linda Koch}

Original article Romão, R. et al. High prevalence of side effects after recombinant human thyrotropinstimulated radioiodine treatment with $30 \mathrm{mCi}$ in patients with multinodular goiter and subclinical/clinical hyperthyroidism. Thyroid 19, 945-951 (2009) 